\title{
Bioequivalence and Bioavailability of Liposomal Doxorubicin - Pharmaco-Kinetic Parameters for Clinical Trials
}

\author{
Tamilselvi Manickam ${ }^{1}$ and Srinivas Ankanagari ${ }^{2 *}$ \\ ${ }^{1}$ Value Added Corporate Services Pvt. Ltd., India \\ ${ }^{2}$ Department of Genetics, Osmania University., India
}

Submission: November 21, 2017; Published: November 28, 2017

*Corresponding author: Srinivas Ankanagari, Department of Genetics, Osmania University, India, Email: srinivasmessage@gmail.com

\section{Introduction}

Bioequivalence study is important for generic drug approval process. In Bioequivalence studies comparison of innovator and reference drug show similar concentration time profile in the blood/plasma of both the rate and extent of absorption of various multisource drug formulations in terms of various bioavailability parameters measured via in vivo testing in human subject. Bio-equivalence cannot be claimed based on in-vitro testing only or on the basis of animal studies. Bio-equivalence of drugs must be determined in humans via established measures of bioavailability. Subsequent batches of the same product are deemed bioequivalent based on in vitro measures such as drug dissolution in a statistically significant manner [1].

The term bioavailability defined as the rate and extent to which the active moiety is absorbed from a drug product and becomes available at the site of action. For drug products which are not absorbed into the bloodstream, bioavailability measurements are needed to measure the rate and extent to which the active moiety becomes available at the site of action. Bioavailability studies focus on determining the process by which a drug is released from the oral dosage form and moves to the site of action. Bioavailability data provides an estimation of the drug absorbed, as well as its subsequent distribution and elimination. Bioavailability can be generally documented by a systemic exposure profile obtained by measuring drug and/or metabolite concentration in the systemic circulation over time. The systemic exposure profile determined during clinical trials in the IND period can serve as a benchmark for subsequent BE studies [2].

Doxorubicin $\mathrm{HCl}$ (doxorubicin) is a widely used chemotherapeutic agent for more than 30 years; it is often given in combination with other drugs. Most serious adverse effects of doxorubicin therapy are decrease in certain types of white blood cells (including neutrophils, which can lead to transient immune-suppression and serious infections), nausea and vomiting, alopecia (hair loss) and damage to the heart muscle. Pegylated Liposomal Doxorubicin (PLD) is a long-circulating liposomal formulation of doxorubicin. This type of liposome is designed to carry doxorubicin, entrapped within its aqueous "core", through the bloodstream and to distribute it to tissues in encapsulated form. PLD liposomes are made to be very small and to circulate within the bloodstream for several days to a week. Long circulation is provided by a layer of polyethylene glycol (PEG) chemically grafted to the liposome surface.

Liposome products target specific tissues; the plasma concentration may not be related to the concentration of drugs at these specific tissues. Method of determining bioequivalence between a reference brand-name drug and a generic drug product for administering to a subject, the method comprises: (a) determining pharmacokinetic parameters of the reference brand-name drug and the generic drug product; (b) determining toxicity associated with administration of the reference brandname drug and the generic drug product; (c) comparing the pharmacokinetic parameters and the toxicity profiles of the reference brand-name drug and the generic drug product; wherein equivalent pharmacokinetic parameters combined with equivalent toxicity profiles of the reference brand-name drug and the generic drug product indicate bioequivalence of the reference brand-name drug and the generic drug product when administered to the subject. Liposomal drug products often pose a challenge in demonstrating bioequivalence between generic and brand name products. After administration, the active pharmaceutical ingredient in a liposomal drug product usually exists in multiple forms in plasma (e.g., free drug and encapsulated 
drug). The products often have physical and chemical properties that critically impact therapeutic effect and safety profile. Many of these critical physicochemical properties need to be characterized by a distribution. Liposomal drug products also exhibit complex in vivo release patterns that are affected by the liposome vesicles, drug substance, and inactive ingredients. Consequently, to demonstrate bioequivalence, it is important to identify the most physiologically relevant analyte(s) for BE purposes, conduct comprehensive, comparative physicochemical testing on a wide range of product properties, and perform in vitro release testing under controlled, physiologically relevant conditions.

Liposomal drug products are often pose a challenge in demonstrating bioequivalence between generic and brand name products. After administration, the active pharmaceutical ingredient in a liposomal drug product usually exists in multiple forms in plasma (e.g., free drug and encapsulated drug). The products often have physical and chemical properties that critically impact therapeutic effect and safety profile. The mechanism of increasing longevity of liposomes in the circulation by steric stabilization has been extensively studied. The ability of PEG to increase the circulation time of the liposomes depends on both the amount of grafted PEG and the length, or molecular weight of the polymer. The greatest improvements in blood residence time were reported with longer-chain PEGs. The purpose of the present study is to compare the various clinical trial studies for the pharmacokinetic parameters.

\section{Methods}

The recent clinical trials, regulatory guidelines of bioequivalence studies on Liposomal Doxorubicin Hydrochloride were considered for the present study for the safe release of Liposomal doxorubicin hydrochloride at the site of cancer cell and the measurement of PK parameters.

\section{Results and Discussion}

In the year of 2000 FDA permits waivers of in vivo bioequivalence testing for Class 1 and also Class 3 drugs. It provided the direction relating to both the solubility and dissolution testing requirements and methods to assess permeability. FDA has determined that in vivo BE testing may not be needed in instances of BCS Class 1 and Class 3 drugs. India also proposed New Requirements for Bio equivalence studies that are to be conducted in India on drugs mainly intended for export. There are numerous phase I and II clinical trials with liposomal formulation. The results from the clinical trials suggest that encapsulation of doxorubicin within a liposome decreases its cardio toxicity and increases its antitumor potency compared to free drug. For the TLC D-99 formulation, the drug is able to accumulate more at sites of tumor growth. The results shown that in vivo the toxicity of a formulation is related to its drug retention properties. Satirically stable liposome's display an increased circulation of drug as well as enhanced antitumor activity. These developed methods for the preparation of liposomal doxorubicin will ultimately help in developing other liposome based anticancer pharmaceuticals [3]. In June 2011, the TGA European guidelines on bioequivalence released more details on the conduct and analysis of bioequivalence studies. Bioequivalence data is mostly considered in applications for new generic products. The assessment of the extent of exposure (area under the curve, or AUC) is typically measured up to the last quantifiable point (AUC0-t) and extrapolated to infinite time (AUC0- $\infty$ ). For drugs with extended half-lives, the sample collection is done for 72 hours. As per TGA, studies conducted under fasting conditions, bioequivalence assessments should be based on levels of the parent drug, even if the parent is an inactive prod rug. Bioequivalence for most products can be determined on only a single highest strength, and stringent limits for narrow therapeutic index drugs (NTI). Paginated Doxorubicin Hydrochloride Liposome compared with the reference product, Doxil@. Show similar physicochemical profile. In addition, it shows bioequivalence to Doxil ${ }^{\circledR}$ in ovarian cancer patients and safe to use [4]. The number of subjects evaluated for PK parameters in bioequivalence studies calculated based on two one sided test procedure conclude the test product is bioequivalent to the reference product [5]. In clinical research Meta-analysis of clinical trial data is an increasingly important method, particularly in the field of therapeutic evaluation [6]. In principle, clinical trials with clinical endpoints are considered necessary to demonstrate therapeutic equivalence. To demonstrate bioequivalence then the $90 \%$ confidence interval range for the ratio test/reference of the $\mathrm{PK}$ parameters of interest should not exceed the upper limit of the acceptance range as described in the guideline on the investigation of bioequivalence [7]. In general, to determine bioequivalence, comparison is done between two products such as a commercially-available Brand-name product and a potential yet to-be-marketed Generic product. Pharmacokinetic studies are conducted whereby each of the preparations are administered in a cross-over study to healthy volunteer subjects, but occasionally in patients. For a pharmacokinetic comparison, the plasma concentration data are used to assess key pharmacokinetic parameters such as area under the curve (AUC), peak concentration (Cmax), time to peak concentration (Tmax), and absorption lag time (tlag). In addition to data from bioequivalence studies, other data may need to be submitted to meet regulatory requirements for bioequivalence. i.e. analytical method validation and in vitro-in vivo correlation studies. The studies have shown, the bioequivalence is met if 90\% confidence interval (CI) of the mean Cmax, AUC (0-t) and AUC $(0-\infty)$ of the pharmaceutically equivalent drug product relative to the reference drug is within $80 \%$ to $125 \%$. In few studies, the bioequivalence determined meets the requirements set by the FDA. Each of the trial results conducted in the past in order to evaluate the efficacy $90 \%$ BE criteria as per the protocol specified [8]. The $90 \%$ confidence interval for the comparison of two drugs lie within an acceptance range of 0.80-1.25. Where as in Cmax-ratio of relative bioavailability may be more variable than 
the AUC-ratio and a wider acceptance range may be acceptable. The range used should be justified in the protocol considering safety and efficacy parameters [9]. To establish bioequivalence, it is important to find physiologically most suitable analyte(s).

Table 1: Statistical analysis of pk parameters for encapsulated and total doxorubicin.

\begin{tabular}{|c|c|c|}
\hline Free Doxorubicinol & Lower Limit & Upper Limit \\
\hline Cmax & 90.83 & 109.85 \\
\hline AUC 0-t & 65.13 & 179.36 \\
\hline AUC 0-inf & 65.29 & 177.02 \\
\hline Total Doxorubicin & Lower Limit & Upper Limit \\
\hline Cmax & 89.38 & 106.01 \\
\hline AUC 0-t & 89.97 & 116.74 \\
\hline AUC 0-inf & 85.70 & 123.35 \\
\hline
\end{tabular}

The above represented statistical analysis (Table 1) revealed that the $90 \%$ confidence interval $(90 \% \mathrm{CI})$ for Log transformed pharmacokinetic parameters are within 80-125 limits for encapsulated and total doxorubicin, while $90 \% \mathrm{CI}$ is not within 80-125 for free doxorubicin and doxorubicinol. The generic liposomal doxorubicin is bioequivalent to the innovator product for encapsulated and total fractions and not bioequivalent for free doxorubicin and doxorubicinol [10]. The 90\% CI of relative bioavailability should lie within an acceptance range of $0.80-1.25$. In case of an especially narrow therapeutic range the acceptance range may need to be tightened. In rare cases (e.g. highly variable drugs) a wider acceptance range may be acceptable if it is based on solid clinical justification. Cmaxratio of relative bioavailability may be more variable than the AUC-ratio and a wider acceptance range may be accepted. The range used should be justified in the protocol taking into account safety and efficacy consideration. Statistical evaluation of Cmax only makes sense if there is a clinically relevant claim for release or action or signs for a relation to adverse effects. The non-parametric $90 \%$ confidence interval for this measure of relative bioavailability should lie within a clinically determined range [11]. Several patents were claimed for liposome associated to free doxorubicin, doxorubicinol and Doxil with proved bioequivalence and bioavailability in plasma sample and the release of active moiety from liposome's at the site of action $[12,13]$. The study findings have shown that efficacy and toxicity profile of the generic drug ((SPIL DXR HCL) is comparable to the reference product DXR HCL liosome injection in fibrosarcoma bearing BALBc mice in terms of antitumor activity, toxicity and pharmacokinetics. Pharmacokinetic bioequivalence shown for Cytori's ATI-0918 liposomal formulation of Doxorubicin Hydrochloride, the outcome of the studies have shown that the pharmacokinetic bioequivalence of ATI-0918 was confirmed as bioequivalent to European sourced CAELYX®. The lower and higher $90 \%$ CI ratios for In-Cmax, ln-AUC0-t and ln-AUC0-inf of free doxorubicin, encapsulated doxorubicin and the metabolite doxorubicinol following ATI-0918 were within 80.00\% to $125.00 \%$ of those ratios following European sourced CAELYX®. ATI-0918 is a complex generic formulation of the, DOXIL ${ }^{\circledR} /$ CAELYX®, which is FDA approved in the U.S. for ovarian cancer, multiple myeloma, and Kaposi's Sarcoma; and in the European Union for breast cancer, ovarian cancer, multiple myeloma, and Kaposi's Sarcoma [14]. The effects of liposome characteristics on oral absorption of doxorubicin, as a hydrophilic lowpermeability drug was investigated. The results have shown that oral absorption is highly dependent on liposomal properties, and optimum formulations are effective for low-permeability drugs [15]. Lipid excipients are critical in the liposome formulation. Lipids should be similar to the reference standard. It is expected to use an active loading process with an ammonium sulfate gradient. An active loading process uses an ammonium sulfate concentration gradient between the liposome interior and the exterior environment to drive the diffusion of doxorubicin into the liposomes. The major steps include 1) formation of liposomes containing ammonium sulfate, 2) liposome size reduction, 3) creation of ammonium sulfate gradient, and 4) active drug loading. Liposome composition including lipid content, free and encapsulated drug, internal and total sulfate and ammonium concentration, histidine concentration, and sucrose concentration should be measured. The drug-to-lipid ratio and the percentage of drug encapsulation can be calculated from liposome composition values [11].

A Bio-IND is required to conduct bioequivalence studies of doxorubicin liposome injection in humans since doxorubicin is a cytotoxic drug. FDA recommended single dose fasting two-way crossover bioequivalence studies in ovarian cancer patients at $50 \mathrm{mg} / \mathrm{m} 2$ dose. Sponsors should measure both liposome-encapsulated and free doxorubicin to demonstrate the same in vivo stability of generic liposome formulation and the reference standard. The studies may be conducted under either fasted or standard diet conditions depending on patient needs [16]. WHO released guidance for the evaluation of generic liposomal formulations in 2013 based on FDA [17]. Doxil® (PE Gylated liposomal doxorubicin) has an increased circulation time compared to free doxorubicin and is up to six times more effective than free doxorubicin. Upon the detachment of PEG coating, membrane destabilization should occur, delivering the liposomal contents to target. Amphipathic drugs (anthracyclines and Vinca alkaloids) appear to be the most suitable for liposomal carriers due to possibility to tune the drug-release rates to maintain the stability of the formulation in the plasma, and to promote the drug release at the tumor site [3]. Many of these critical physicochemical properties need to be characterized by a distribution. Liposomal drug products also exhibit complex in vivo release patterns that are affected by the liposome vesicles, drug substance, and inactive ingredients. Consequently, to demonstrate bioequivalence, it is important to identify the most physiologically relevant analyte(s) for BE purposes, conduct comprehensive, comparative physicochemical testing on a wide 
range of product properties, and perform in vitro release testing under controlled, physiologically relevant conditions. Liposomes have acted as carriers of a choice for drug and gene delivery. Such a delivery system should clearly show benefit of fast and effective accumulation in target tumors, higher drug delivery in tumors than other drug delivery systems and ability to internalize by the target cells thus creating high intracellular drug concentration. It is also desirable to use internalizable ligands to facilitate delivery of liposomal drug inside cells followed by release of drug to achieve the desired therapeutic effect for a reasonable period of time [4].

\section{Conclusion}

In conclusion, the comparison of bioequivalence studies on Liposomal Doxorubicin- Hydrochloride shows significant advantages of using liposomes as a career vehicle which reduced cardio toxicity and have enhanced antitumor effect in various cancers

\section{References}

1. http://commercialeyes.com.au/tgas-updated-guideline-forevaluation-of-bioequivalence-data/

2. Schug B, Donath F (2012) Phase-I-studies with newly developed liposomal studies with newly developed liposomal formulations: safety and efficacy issues. Socra Tec R\&D.

3. Xing M, Yan F, Yu S, Shen P (2015) Efficacy and cardiotoxicity of liposomal doxorubicin-based chemotherapy in advanced breast cancer: a meta-analysis of ten randomized controlled trials. PLoS One 10(7): e0133569.

4. Sawant RR, Torchilin VP (2012) Challenges in development of targeted liposomal therapeutics 14(2): 303-315.

5. (2015) Louise Zornoza, Reg Link News.
6. Ali SM, Sheikh S, Ahmad A, Ahmad MU, Chen P, et al. (2016) Bioequivalence study of pegylated doxorubicin hydrochloride liposome (pegadria) and doxil® in ovarian cancer patients: physicochemical characterization and pre-clinical studies. International Journal of Nanomedicine \& Nanotechnology.

7. https://clinicaltrials.gov/ct2/show/NCT02260544

8. Cucherat M, Boissel JP, Leizorovicz A, Haugh MC, Easy MA, et al. (1997) A program for the meta-analysis of clinical trials. Comput Methods Programs Biomed 53(3): 187-190.

9. (2004) Committee for Medicinal Products for Human Use. European medicines Agency.

10. Simonian DR, Laird N (1986) Meta-analysis in clinical trials. Control Clin Trials 7(3): 177-188.

11. Zheng N, Jiang W, Lionberger R, Yu LX (2014) Bioequivalence for liposomal drug products. In: yu l, li b (eds.), fda bioequivalence standards. AAPS Advances in the Pharmaceutical Sciences Series,13 Springer, New York, USA.

12. Venkateswarlu K, Thirumara J (2013) Review on bioavailability and bioequivalence studies. International Journal Pharmacy Science Rev Res 18(1): 56-64.

13. Patents methods for determining liposome bioequivalence, USA.

14. Crommelin DJA (2012) Comparative in vitro characterization of liquid characterization of liquid liposomal formulations.

15. (2017) Cytori Reports Bioequivalence Trial Results in Ovarian Cancer at AAPS.

16. Daeihamed M, Haeri A, Ostad SN, Akhlaghi MF, Dadashzadeh S, et al. (2017) Doxorubicin-loaded liposomes: enhancing the oral bioavailability by modulation of physicochemical characteristics. Nanomedicine 12(10):1187-1202.

17. Gaspani S, Milani B (2013) Access to liposomal generic formulations: beyond AmBisome and Doxil/Caelyx. Generics and Biosimilars Initiative Journal (GaBI Journal) 2(2): 60-62.

Your next submission with Juniper Publishers
will reach you the below assets
- Quality Editorial service
- Swift Peer Review
- Reprints availability
- E-prints Service
- Manuscript Podcast for convenient understanding
- Global attainment for your research
- Manuscript accessibility in different formats
( Pdf, E-pub, Full Text, Audio)
- Unceasing customer service
Track the below URL for one-step submission
https://juniperpublishers.com/online-submission.php

